\title{
A proposed five kilo-ton Cherenkov scintillation detector at CJPL
}

\author{
Wentai Luo \\ University of Chinese Academy of Sciences \\ Jinping Neutrino Experiment \\ E-mail: luowentail5emails.ucas.ac.cn
}

We propose to build a Cherenkov scintillation detector with five kilo-ton target mass in the China Jinping Underground Laboratory (CJPL). The deepest vertical overburden and the longest distance to any commercial nuclear power plants enable us to carry out advanced low-energy neutrino experiments for solar neutrino, geo-neutrino and supernova neutrino physics studies. Simulation studies have been done on the physics sensitivities and background sources. We have made progress on the development of the slow scintillation technique and the optimization of the detector geometry. Results on a prototype detector running in CJPL are also presented.

XXIX International Symposium on Lepton Photon Interactions at High Energies - LeptonPhoton2019 August 5-10, 2019

Toronto, Canada 


\section{Introduction}

China JinPing underground Laboratory (CJPL) is one of the ideal sites to do low background experiments in the world because of $2,400 \mathrm{~m}$ rock overburden. A multi kiloton detector is proposed to measure low-energy neutrinos, such as solar neutrino, geoneutrino, supernova neutrino, etc. The China Jinping Underground Laboratory(CJPL) [2] is located in Sichuan province, China, and 2,400 meters under Jinping mountain, southwestern region of China. It is about a 5 hour trip from Beijing to CJPL ( 3 hours direct flight from Beijing to Xichang +2 hours drive from Xichang to CJPL). The $2,400 \mathrm{~m}$ vertical rock overburden means that cosmic-ray muon induced radioactive isotopes, which are a very serious background for low-energy neutrino events, are expected to be significantly suppressed at Jinping. Jinping is also far away from all the nuclear power plants in operation and under construction. The closest reactor is about $950 \mathrm{~km}$ away. Therefore CJPL has the lowest reactor neutrino and cosmic ray muon background. A comparison with other underground labs can be seen in Fig.1[1].

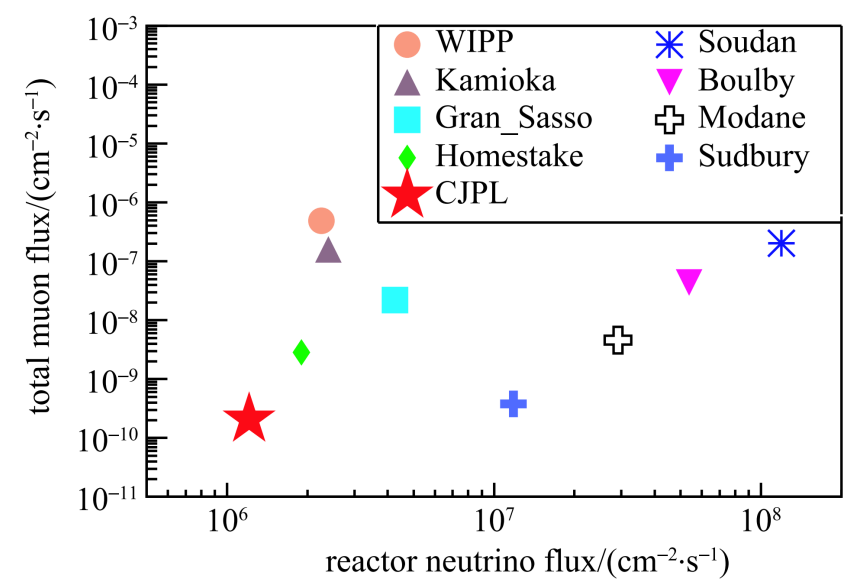

Figure 1: Muon flux vs reactor neutrino background flux for various underground labs in the world.

\section{Slow liquid scintillator}

Slow liquid scintillator detector could separate Cherenkov light and scintillation by space and time, as shown in Fig.2. One can reconstruct the energy and direction of a point-like vertex simultaneously. In this study, samples of LAB with around $0.07 \mathrm{~g} / \mathrm{L}$ of PPO and $13 \mathrm{mg} / \mathrm{L}$ of bis-MSB or formulas with similar concentrations are shown to display a good balance between scintillation decay time and light yield. This combination with the PMT detection approach could serve as good slow liquid scintillator candidates for solar and supernova relic neutrino experiments. The concentrations of PPO and bis-MSB could be further optimized by using a large test apparatus and by performing extensive off line simulations and analyses[2]. 


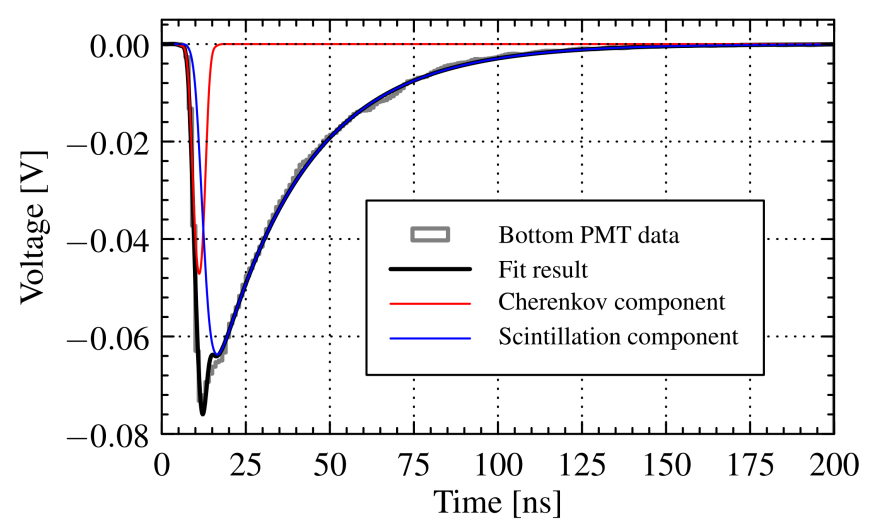

Figure 2: The fit to the waveforms of the PMT for the sample of LAB with $0.07 \mathrm{~g} / \mathrm{L}$ PPO and $13 \mathrm{mg} / \mathrm{L}$ bis-MSB.

\section{One-ton prototype current status}

A one-ton prototype is designed and running in CJPL to test the performance of several related key detector components, to understand the neutrino detection technology, and to measure the underground background level. A total of 30 Hamamatsu R5912 PMTs and $1.2 \mathrm{~m}^{3}$ water or slow liquid scintillator $(\mathrm{LAB}+0.07 \mathrm{~g} / \mathrm{L} \mathrm{PPO}+13 \mathrm{mg} / \mathrm{L}$ bis-MSB$)$ are utilized in this prototype. Water phase is from May 08th, 2017 to July 26th, 2017, liquid Scintillator (LS) phase from July 31th, 2017 to now. In ${ }^{214} \mathrm{Bi}$ cascade decay measurement, ${ }^{214} \mathrm{Po}$, which is the daughter of ${ }^{214} \mathrm{Bi}$ has a very short half-life. So the $\beta$ from ${ }^{214} \mathrm{Bi}$ and $\alpha$ from ${ }^{214} \mathrm{Po}$ can form a cascade signal. Assuming all later ${ }^{214} \mathrm{Bi}$ from ${ }^{238} \mathrm{U}$ (90 days after beginning), considering the current background level, the ${ }^{238} \mathrm{U}$ detection limit reaches $10^{-15} \mathrm{~g} / \mathrm{g}$. And we also found Radon diffusion. The deviation of ${ }^{214} \mathrm{Bi}$ vertex distribution in Z-axis from origin indicates that air leaks from top filling and instrument pipes. LS purification and equipment upgrade is running. From July 13th we began to fill nitrogen into our detector, increasing the inner pressure of detector in order to stop Radon coming in, and we hope such decrease continues. Several muon events were also detected. The angular distribution of these muons are also reconstructed.

\section{Five kilo-ton detector in the future}

In the future, we propose to build a Cherenkov scintillation detector with kilo-ton target mass in the China Jinping Underground Laboratory (CJPL). The optimization of detector structure is based on a simulation of radioactive background. Neutrino signals in the fiducial volume should be much more than the external background, which will be reduced by an order of magnitude behind a 0.5 -meter water shield. ${ }^{214} \mathrm{Bi}$ and ${ }^{208} \mathrm{Tl}$ are the main sources of background owing to the high energy $(>2 \mathrm{MeV}) \gamma$ emission and 5 kilo-ton detector structure is shown in Fig.3.

We use maximize likelihood method to reconstruct the recoil electron direction, which is shown as: 


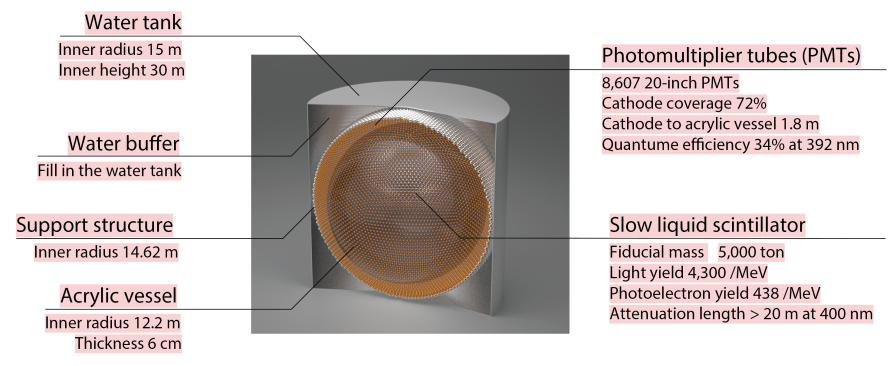

Figure 3: 5kt detector structure and some setup in simulation

$$
\log L\left(n_{i}, t_{i j}, E, x, y, z, t, \theta, \phi\right)=\sum_{i=1}^{N} \log P_{i}^{C}+\sum_{i=1}^{N} \sum_{j=0}^{n_{i}} \log P_{i j}^{T}
$$

In this function, $n_{i}$ is PE number of the ith PMT, $t_{i j}$ is the time of $j$ th PE on the ith PMT, $E, x, y, z, t, \theta, \phi$ are the energy, position, time and direction of the vertex, $P_{i}^{C}$ is the PE number probability density function of the ith PMT, $P_{i j}^{T}$ is the PE time probability density function of jth PE on the ith PMT. We use $5 \mathrm{kt}$ detector to simulate $2 \mathrm{MeV}$ recoil electrons in the center. Angle between fitted direction and truth is shown in Fig.4. The peak is about 20 degrees from likelihood reconstructed results, and about $84 \%$ of the events lie in 90 degree by using waveform likelihood method.

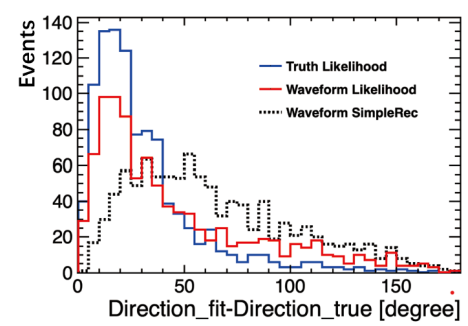

\begin{tabular}{|llll}
\hline Item & Method & Dataset & Initial value \\
\hline $\begin{array}{l}\text { Truth } \\
\text { Likelihood }\end{array}$ & $\begin{array}{l}\text { Maximize } \\
\text { likelihood }\end{array}$ & PE truth & Generator truth \\
$\begin{array}{l}\text { Waveform } \\
\text { Likelihood }\end{array}$ & $\begin{array}{l}\text { Maximize } \\
\text { likelihood }\end{array}$ & Waveform & Generator truth \\
$\begin{array}{l}\text { Waveform } \\
\text { SimpleRec }\end{array}$ & Barycenter & Waveform & \\
\hline
\end{tabular}

Figure 4: $2 \mathrm{MeV}$ electrons directional reconstruction results compared with truth direction

\section{Conclusion}

A one-ton prototype detector has been running at CJPL since July 2017. From the observed ${ }^{214} \mathrm{Bi}$-decay chain, we have studied the detector performance, radon background and cosmic ray muons. We investigated various radioactive background sources in materials and performed a customized Geant4 simulation study to optimize the design of a five-kiloton neutrino detector with slow scintillator. Slow liquid scintillator could separate the prompt Cherenkov light from the potential scintillation light with longer emitting time. We applied a time profile analysis together with charges from both the fired and unfired photo-tubes to simultaneously reconstruct the vertex, energy and direction in the detector. 


\section{References}

[1] John F. Beacom, Shaomin Chen et al, Physics prospects of the Jinping neutrino experiment, Chinese Physics C 41, 023002 (2017).

[2] Ziyi Guo, Minfang Yeh et al, Slow liquid scintillator candidates for MeV-scale neutrino experiments, Astroparticle Physics 109 (2019): 33-40. 